\title{
Aortic Proximalisation - Zone 0 vs. Zone 2. A Concept or True Challenge?
}

\author{
Cian Tan ${ }^{1}$, Aleksandra Lopuszko ${ }^{1}$, Wahaj Munir ${ }^{1}$, Mohamad Bashir ${ }^{2}$, and Benjamin \\ Adams $^{3}$ \\ ${ }^{1}$ Queen Mary University of London Barts and The London School of Medicine and \\ Dentistry \\ ${ }^{2}$ NHS Wales Health Education and Improvement Wales \\ ${ }^{3}$ Barts Health NHS Trust
}

May 25, 2021

\begin{abstract}
Background Use of the Frozen Elephant Trunk (FET) device to manage complex surgical pathologies of the aorta (e.g. acute Type A aortic dissection) has gained popularity since its introduction in the early 2000s. Though the distal anastomosis was traditionally performed at Zone 3 (Z-3-FET), preference gradually shifted towards Zone 2 (Z-2-FET) in favour of improved surgical access and outcomes. This review seeks to elucidate whether proximalisation of arch repair to Zone 0 (Z-0-FET) would further improve postoperative outcomes. Methods We performed a review of available literature to evaluate the comparative efficacies of Z-2-FET versus Z-0-FET, in terms of surgical technique, clinical outcomes, and incidence of adverse events. Results Z-0-FET seems to be associated with a more accessible surgical approach, and shorter cardiopulmonary bypass, antegrade cerebral perfusion, and cardioplegia durations than Z-2-FET. Further, Z-0-FET is could potentially be associated with a lower incidence of neurological, renal, and recurrent laryngeal nerve injury, as well as mortality and reintervention rates than Z2-FET. This said, Z-0-FET is itself associated with significant challenges, and efficacy in terms of postoperative true lumen integrity and false lumen thrombosis is mixed. Conclusion Current literature seems to suggest that Z-0-FET procedures are more straightforward and associated with lower rates of certain adverse events, however, the majority of data reviewed is retrospective. This review therefore recommends prospective research into the comparative strengths and limitations of Z-0FET and Z-2-FET to better substantiate whether proximalisation of arch repair represents a concept, or a true challenge to advance surgical intervention for arch pathologies.
\end{abstract}

AORTIC PROXIMALISATION - ZONE 0 VS ZONE 2.

A CONCEPT OR TRUE CHALLENGE?

Running Head: Aortic Arch Repair Proximalisation

Sven Zhen Cian Patrick Tan ${ }^{1} \mid$ Aleksandra Lopuszko ${ }^{1}$ | Wahaj Munir ${ }^{1}$

Benjamin Adams FRCS ${ }^{2}$ | Mohamad Bashir MD, PhD, MRCS $^{3}$

1: Barts and The London School of Medicine and Dentistry, Queen Mary University of London, E1 2AD, United Kingdom

2: Department of Cardiothoracic Surgery, St Bartholomew's Hospital, London, EC1A 7BE, United Kingdom

3: Vascular \& Endovascular Surgery, Health Education \& Improvement Wales, Wales, UK

\section{Correspondence:}


Mohamad Bashir M.D. Ph.D. MRCS ${ }^{3}$

drmobashir@outlook.com

Vascular and Endovascular Surgery,

Health Education \& Improvement Wales

Wales, UK

Funding declarations: None to be declared

Conflicts of interest: None to be declared

Data availability statement: Data sharing is not applicable to this article as no new data were created or analysed in this study.

Keywords: Aortic arch, arch repair, aortic dissection, frozen elephant trunk, Zone 0, Zone 2

\section{ABSTRACT}

\section{Background}

Use of the Frozen Elephant Trunk (FET) device to manage complex surgical pathologies of the aorta (such as acute Type A aortic dissection) has gained popularity since its introduction in the early 2000s. Though the distal anastomosis was traditionally performed at aortic Zone 3 (Z-3-FET), preference gradually shifted towards Zone 2 (Z-2-FET) in favour of improved surgical access and clinical outcomes. This review seeks to elucidate whether proximalisation of arch repair to Zone 0 (Z-0-FET) would further improve postoperative outcomes.

\section{Methods}

We performed a review of available literature to evaluate the comparative efficacies of Z-2-FET versus Z-0FET, in terms of surgical technique, clinical outcomes, and incidence of adverse events.

\section{Results}

Z-0-FET seems to be associated with a more accessible surgical approach, and shorter cardiopulmonary bypass, antegrade cerebral perfusion, and cardioplegia durations than Z-2-FET. Further, Z-0-FET is could potentially be associated with a lower incidence of neurological, renal, and recurrent laryngeal nerve injury, as well as mortality and reintervention rates than Z-2-FET. This said, Z-0-FET is itself associated with significant challenges, and efficacy in terms of postoperative true lumen integrity and false lumen thrombosis is mixed.

\section{Conclusion}

Current literature seems to suggest that Z-0-FET procedures are more straightforward and associated with lower rates of certain adverse events, however, the majority of data reviewed is retrospective. This review therefore recommends prospective research into the comparative strengths and limitations of Z-0-FET and Z2-FET to better substantiate whether proximalisation of arch repair represents a concept, or a true challenge to advance surgical intervention for arch pathologies.

\section{BACKGROUND}

Structural pathologies of the aortic arch and descending thoracic aorta (DTA) are notoriously challenging to manage via surgical intervention and are renowned for being associated with high mortality and postoperative disability rates, as well as high cost. ${ }^{1}$ Historically, pathologies such as aortic aneurysm and De Bakey Types I and II (Stanford Type A) aortic dissection warranted multiple interventions and admissions, and were associated with relatively poor clinical outcome. ${ }^{2}$ In an effort to simplify surgical repair of the aortic arch, the two-stage conventional Elephant Trunk (cET) procedure was introduced in 1983 by Borst et al . It involved an initial total arch replacement (TAR) via median sternotomy, followed by implantation of an 
elephant trunk graft in a second procedure (Figure 2). ${ }^{3}$ Borst and colleague's technique enjoyed widespread use until the introduction of the Frozen Elephant Trunk (FET) approach to aortic repair by Haverich et al. in 2003, which replaced the free-floating elephant trunk prosthesis introduced by Borst et al. with a secured stent inserted into the descending thoracic aorta. ${ }^{4}$ The FET was heralded for reducing procedure duration, and rates of postoperative complications associated with cET, indeed over 28,000 FET procedures were carried out between its advent and 2014. ${ }^{5}$ TAR with FET is currently indicated for repairing aneurysms of the aortic arch and DTA, as well as acute, chronic, and residual Type A aortic dissection. ${ }^{5}$

When faced with a Type A aortic dissection, the aortic surgeon is presented with the option to proceed either a conservative or aggressive therapeutic approach. ${ }^{6,7}$ Whereas the former advocates the use of an initial hemiarch repair (HAR) before taking further steps to address the sequalae of Type A aortic dissection, the latter recommends a TAR with deployment of an FET to control intimal tears and stimulate false lumen (FL) remodelling. ${ }^{8}$ The debate between conservative and aggressive intervention is ongoing - Bashiret al. note that this is in part due to the lack of prospective studies comparing the two. ${ }^{8}$ Studies by Rice et al. and Sun et al. have produced results that seem to suggest there is no significant difference in perioperative mortality between HAR and TAR procedures for Type A aortic dissection, and Bashir et al. highlight that more premptive, aggressive approaches could be associated with higher rates of 5-year freedom from death, rupture, and reintervention. ${ }^{8-10}$ It should also be noted that TAR not only reduces the risk of further aortic dilation, but is also able to fully obliterate the distal FL. ${ }^{10}$

Apart from total versus hemiarch replacement, the surgeon must also consider the primary differences between cET and FET prostheses deployed for arch repair. Whereas cET arch repair introduced a free-floating aortic graft into the DTA true lumen (TL), anastomosed to the distal end of an aortic arch graft, the FET procedure uses endovascular stent secured to the native aortic intima and anastomosed to a Dacron arch prosthesis. $^{3}$

Although use of the FET is advantageous over cET as it involves only one surgery and is associated with fewer complications and a lower re-intervention rate, it remains a technically demanding intervention. ${ }^{5}$ The complications associated with FET are especially debilitating - spinal cord injury, cerebral injury, and kidney failure are cited as occurring in up to $11 \%, 26 \%$, and $22 \%$ of cases respectively. ${ }^{11,12}$

Notably, the aortic zone at which the elephant trunk stent is anastomosed to the aortic arch graft is a key issue of debate. ${ }^{1}$ When Haverich and colleagues introduced the FET procedure, distal anastomosis at Zone 3 (Z-3-FET) seemed to be the conventional approach. However, in recent decades, this technique has given way to distal anastomosis at Zone 2 (Z-2-FET) - which is now the preferred surgical approach as it is associated with even better clinical outcomes than Z-3-FET. ${ }^{5}$ Following this paradigm shift, surgeons have began questioning whether proximalising FET implantation to Zone 0 (Z-0-FET) would further improve surgical operability and clinical outcomes. ${ }^{1}$ This begs the question: is proximalisation of aortic repair from Zone 2 to Zone 0 simply a concept, or true challenge?

Therefore, this review seeks to evaluate current literature and compare Z-2-FET and Z-0-FET in terms of surgical technique, clinical efficacy, and incidence of key complications (mortality, neurological injury, renal injury, recurrent laryngeal nerve injury, and need for reintervention).

\section{ZONE 2 VS ZONE 0: SURGICAL TECHNIQUE}

In assessing whether proximalisation of aortic arch repair with FET truly represents advancement, the surgical approaches for Zone 0 and Zone 2 arch repair should be compared. Though surgical techniques for both approaches vary between centres and devices, the procedure can be summarised as involving four main phases: initiation, aortotomy, prosthesis implantation, and rewarming. ${ }^{5}$ Techniques for Zone 2 FET arch repair are widely documented and are broadly similar to Zone 3 arch repair (Figure 1). ${ }^{5,13,14}$ In contrast, arch repair can be achieved via two main approaches in Zone 0 FET, depending on the prosthetic device employed. These are examined below.

Initiation 
Both approaches begin with a median sternotomy and initiation of cardiopulmonary bypass (CPB), with the axillary, innominate, or femoral arteries used as sites for systemic perfusion and the superior and inferior vena cava used for venous outflow. ${ }^{13-16}$ A guidewire into the aortic true lumen is also sometimes introduced via the femoral artery at this point. ${ }^{13}$ Subsequently, hypothermic circulatory arrest (HCA) and selective anterograde cerebral perfusion (ACP) are established. ACP can be provided via the brachiocephalic trunk and left carotid artery, or via the carotid arteries directly in cases where the brachiocephalic trunk is cannulated for CPB. ${ }^{5}$ Myocardial protection can be facilitated via cold crystalloid cardioplegia (via a single infusion) or cold blood cardioplegia (infusions at 20-minute intervals). Aortic repair typically begins once the patient is cooled to a core temperature of $25^{\circ} \mathrm{C} .{ }^{11-13,16-18}$ An alternative, branch-first, approach described by Matalanis et al., which can potentially avoid deep hypothermia and circulatory arrest altogether can also be taken. ${ }^{19}$ Following medial sternotomy and initiation of $\mathrm{CPB}$ via both femoral and right axillary arteries, the brachiocephalic trunk is clamped and transected. It is then anastomosed to the first branch of a trifurcated Spielvogel graft (a trifurcated graft introduced by Spielvogel et al . consisting of a single, vertical graft with two shorter lateral branches, that facilitates anastomosis of the arch vessels to an arch graft proximal to the sinotubular junction). ${ }^{20} \mathrm{~A}$ similar approach is taken with the left common carotid, which is anastomosed to the second branch, while cerebral perfusion via the right common carotid is restored. Similarly, perfusion through the left common carotid is restored prior to clamping, resection, and anastomosis of the left subclavian. ${ }^{20}$ Therefore, cerebral, cardiac, and distal perfusion is not interrupted at all during arch vessel anastomosis. Following successful arch vessel anastomosis, the arch is resected to the desired extent and the arch graft introduced. ${ }^{11,16,20}$

\section{Aortotomy}

With cardioplegia and ACP successfully established, aortic resection is performed to facilitate the introduction of the FET stent graft, and subsequently, the proximal aortic graft. In Z-2-FET, the aortic arch is fully resected from the sinotubular junction to the proximal Zone 3 border, and the descending thoracic aorta (DTA) is then examined via angioscope. ${ }^{14,16}$ However, for Z-0-FET, an aortotomy is usually performed up to $15 \mathrm{~mm}$ distal to the sinotubular junction and 10-20 $\mathrm{mm}$ proximal to the origin of the brachiocephalic trunk, allowing the removal of a significant portion of the ascending aorta. Subsequently, both Yamamoto et al. and Jakob et al.reapproximate the walls of the remaining proximal ascending aorta with surgical glue and staying sutures. ${ }^{16,17}$ Aortic valve resuspensions can also be performed to preserve valve function. ${ }^{17}$

\section{Prosthesis implantation and rewarming}

Following resection of the aortic arch, the FET stent graft is then implanted and anastomosed. The guidewire preoperatively placed in the thoracic aorta is used to aid anterograde introduction of the FET stent graft which, when positioned, is anastomosed with the native aortic wall and proximal arch graft at Zone 2, at the distal border of the left subclavian artery. ${ }^{11,}{ }^{13}$ Lower body perfusion is initiated following distal stent to graft anastomosis, usually via a $4^{\text {th }}$ branch in the arch graft in devices such as Thoraflex, E-vita Hybrid Plus, and Frozenix J Graft. ${ }^{11-13}$ Alternatively, in systems lacking a fourth branch (such as E-vita Open), lower body perfusion is achieved via insertion of a Foley catheter into the graft lumen. ${ }^{5}$ In addition, Desai and colleagues also suggest combining TAR with a thoracic endovascular aortic repair (TEVAR) graft anastomosed at Zone $2 .{ }^{21}$ A 2-branched Vascutek Gelweave arch graft is implanted following aortotomy, and anastomosis of the left common carotid and brachiocephalic trunk follows. The graft is proximally anastomosed to an ascending aortic graft, and the TEVAR single-branched stented endograft (GORE TBE) is then introduced via a guidewire introduced at the femoral artery and externalised at the left brachial artery. Stent positioning and rotational alignment is confirmed, and the portal branch is secured in the root of the left subclavian. ${ }^{21}$ Once proximal graft anastomosis is completed, the arch vessels are reimplanted onto the arch graft. Rewarming and reperfusion are then initiated. ${ }^{5}$

Graft implantation in Z-0-FET using the Frozenix J Graft and Thoraflex systems, described by Yamamoto et al. and Jiang et al.respectively, is similar to Z-2-FET. ${ }^{16,18}$ The FET is advanced anterograde into the descending aorta following aortotomy, and the distal end of the stent is positioned superior to level of the aortic valve. The stent is then anastomosed to the four-branched polyester graft and the native aortic 
wall at the distal zone 0 border, and lower body perfusion is initiated via the fourth branch. Following proximal anastomosis, the arch vessels are then reimplanted: the brachiocephalic trunk and left carotid arteries are anastomosed to the second and third graft branches respectively, and the left subclavian is eventually anastomosed to the first branch. The fourth branch is ligated (Figure 2). ${ }^{16,18}$

An alternative approach to Z-0-FET, using the 3-zone (not to be confused with zone 3) E-novia prosthesis, was first outlined by Jakob et al. in May 2020 (Figure 3). ${ }^{17}$ This novel approach deploys a single, continuous prosthesis divided into three zones: a proximal polyester cuff used to secure the zone 0 anastomosis, an uncovered stent portion to span the aortic arch, and finally a distal covered stent (elephant trunk) portion positioned in the proximal descending aorta. ${ }^{17}$ Following aortotomy and aortic root repair, the resected proximal ascending aorta is replaced with a regular tube graft. The 3-zone graft is then introduced anterograde along the guidewire into the descending aorta and positioned such that the proximal end of the elephant trunk portion is positioned just distal to the origin of LSA. The non-covered stent portion is then manually moulded to the curvature of the aortic arch and secured with a staying suture. Finally, the proximal collar is trimmed and anastomosed to the distal end of the ascending aortic prosthesis. Rewarming is then initiated. ${ }^{17}$ Notably, the aortic arch is left relatively untouched: the non-covered stent portion provides structural integrity while facilitating blood flow out of the three arch vessels.

Proximalisation of the FET procedure from zone 3 to zone 2 has already shown to provide improved surgical access and facilitate a more straightforward surgical approach. ${ }^{1,5}$ By bringing the anastomosis forward into the surgical field, the surgeon is afforded easier access to the aortic arch - it therefore stands to reason that proximalisation from zone 2 to zone 0 would further amplify these advantages and further simplify an already exceedingly complex procedure. ${ }^{5}$ Indeed, the difficulty in accessing the proximal DTA via midline sternotomy should not be understated. This would have the added benefit of reducing overall surgical trauma, overall duration of the procedure, and hence time spent under $\mathrm{CPB}, \mathrm{ACP}$, and $\mathrm{HCA}$ - factors which are welldocumented indicators of poor postoperative prognosis. ${ }^{18}$ Indeed, Yamamoto et al. and Jakob et al. highlight the mean CPB duration for Z-0-FET as being $184( \pm 34)$ minutes and $176( \pm 39)$ minutes respectively, compared to $262( \pm 84)$ minutes and $254( \pm 52)$ minutes reported for Z-2-FET by Beckmann et al. and Jakob and colleagues. ${ }^{11,12,16,17}$ Similarly, HCA duration for Z-0-FET is cited as being around $47( \pm 7)$ minutes, compared to up to $126( \pm 43)$ minutes for Z-2-FET. ${ }^{11,16}$ At this juncture, it is also worth emphasising that anastomosis of the arch vessels to a trifurcated graft in Z-0-FET (e.g., the Spielvogel device) is itself challenging, as is the proximal graft-aorta anastomosis. ${ }^{20}$ Sinusoidal orientation of the trifurcated graft, and supra-aortic stenosis at the level of the graft take-off, are possible intraoperative complications to be dealt with - perhaps these could be avoided by performing proximal anastomosis at Zone 1 .

Choudhury et al. outline the features, benefits, and drawbacks of several key prosthetic systems available for aortic arch repair with FET. ${ }^{5}$ Notably, the E-vita Hybrid Plus and Frozenix J Graft prostheses both feature a two-stage non-stented graft and stented-FET design, allowing deployment from either zone 2 or $0 .{ }^{16,22}$ Further, the intussuscepted design of the E-vita Hybrid Plus system also allows deployment from zones 3 and $4 .{ }^{23}$ Indeed, Harky et al. suggest that the E-vita Hybrid Plus is associated with lower rates of postoperative mortality than the Thoraflex device, which Jiang et al. concluded constitutes a viable surgical bailout option when used in Z-0-FET repairs. ${ }^{18,} 24$ These systems therefore offer a more patient-centric approach, specific to the unique anatomy and surgical context of each case and are perhaps best suited to facilitating proximalisation of aortic arch repair.

\section{ZONE 2 VS ZONE 0: CLINICAL OUTCOMES}

Having evaluated the difference in surgical approach between Z-2-FET and Z-0-FET, the comparative efficacies of both procedures should be analysed in terms of postoperative outcome. The improved clinical outcomes associated with proximalisation from Z-3-FET to Z-2-FET have been widely documented, and indeed this has spurred a general shift in preference towards Z-2-FET over the last few years. ${ }^{1,5,24}$ Z-2-FET has been demonstrated to yield fewer long-term adverse events, such as mortality, spinal cord injury, visceral ischaemia, re-intervention, and renal complications, than Z-3-FET. ${ }^{1,5}$ How, then, does Z-0-FET compare to Z-2-FET? 


\section{False lumen thrombosis and aortic remodelling}

The extent of FL thrombosis and positive aortic remodelling following FET implantation can be considered an indicator of therapeutic efficacy of the FET stent. Coverage and rectification of intimal tears in AAD serve to improve distal TL integrity and limit FL patency, thereby promoting thrombosis and obliteration, particularly in the locality of the FET stent. ${ }^{25}$ In cases of chronic aortic dissection, proximal FL thrombosis around the stented aorta is associated with improved distal positive remodelling, while negative aortic remodelling is associated with reintervention in the distal aorta. ${ }^{25}$ Jakob and colleagues, in their review of the E-vita Open hybrid graft for Z-2-FET and Z-3-FET, report complete exclusion of aortic disease and positive remodelling around the graft region in $94 \%(\mathrm{n}=167)$ and $92 \%(\mathrm{n}=163)$ of AAD patients treated with Z-2-FET, but that $82 \%(n=145)$ of such patients were also found to have residual disease distal to the FET stent. ${ }^{11}$ Interestingly, similarly mixed results were reported by both Yamamotoet al. and Jakob et al. in their respective reviews on Z-0-FET. ${ }^{16,17}$ Jakob and colleague's 3-zone Z-0-FET prosthesis procedure showed thrombosis of the FL around the aortic arch and DTA in only $40 \%(n=2)$ and $60 \%(n=3)$ of cases respectively, while 3 of 6 patients reviewed demonstrated residual arch FL patency while remaining clinically stable. ${ }^{17}$ Yamamoto et al.highlighted that thrombosis of FLs of the aortic arch, DTA, and abdominal aorta was achieved in $74.1 \%$ $(\mathrm{n}=80), 29.6 \%(\mathrm{n}=32)$, and $5.5 \%(\mathrm{n}=6)$ of cases respectively. ${ }^{16}$ Interestingly, $3 \%(\mathrm{n}=3)$ patients demonstrated re-opening of the FL around the coeliac arteries following Z-0-FET repair. ${ }^{16}$ Undoubtedly, the clinical factors surrounding the effect of FET on FL thrombosis and aortic remodelling is complex and multifactorial - yet Yamamoto and colleague's results are promising, particularly for cases of DeBakey Types I and II AAD.

\section{Mortality}

In-hospital and 30-day mortality are key considerations surrounding any form of surgery - but are particularly relevant in such a high-risk, complex procedure as aortic arch replacement. Interestingly, findings from Yamamoto et al. and Jakob et al. regarding mortality following Z-0-FET seem to suggest proximalisation of aortic arch repair does indeed represent advancement, especially when compared to results on postoperative mortality from Leone et al. , Tsagakis et al., Jakob et al., and Beckmann et al. Yamamoto and colleagues report a $6.5 \%(n=7)$ rate of in-hospital mortality, attributable to multisystem failure $(n=3)$, heart failure $(n=1)$, exacerbation of pre-existing cerebral infarct $(n=1)$, pneumonia $(n=1)$, and left ventricular rupture $(\mathrm{n}=1) .{ }^{16}$ Jakob et al. reported a single in-hospital mortality (their only mortality to date) attributable to right heart failure following Z-0-FET. ${ }^{17}$ In contrast, Z-2-FET seems to be associated with in-hospital mortality rates between $11 \%$ and $20 \%$, attributable to factors including cardiogenic shock, heart failure, aortic rupture, pulmonary failure, pulmonary embolism, and ischaemic cerebrovascular accident. ${ }^{11-13,22,23}$ A similar trend is observed in 30-day mortality rates: while Yamamoto et al. reports a 1.8\% rate of 30-day mortality and Jakob et al. notes that the 5 surviving patients reviewed survived for between 4 to 38 months post-Z-0-FET repair, Tsagakis et al. , Beckmann et al. and Jakob et al. note 30-day mortality rates of 11\%, $8 \%$, and $10 \%$ respectively. ${ }^{11-13,} 16,23$ At this stage, because the Z-0-FET approach is still relatively novel, it is unclear whether this discrepancy is due to the Z-0-FET approach being truly superior to Z-2-FET repair. Yet, it is worth noting that at baseline, the patients included in the above-mentioned trials are relatively heterogenous and similar to one another both clinically and aetiologically.

\section{Neurological injury}

Neurological injury is a common and well-documented complication associated with aortic arch repair. Spinal cord ischaemia (SCI) can occur in up to $7.3 \%$ of cases, while cerebrovascular accidents occur in up to $15.9 \%$ of cases. ${ }^{5}$ A meta-analysis by Bashiret al. reports a pooled estimate of incidence of $7 \%$ and $3.5 \%$ for CVA and paraplegia respectively. ${ }^{1}$ Intriguingly, Yamamoto and colleagues reported no cases of SCI or CVA following Z-0-FET, while the single mortality in Jakob and colleagues' report was attributed to cerebrovascular insufficiency. ${ }^{16,17} 50 \%(\mathrm{n}=3)$ patients experienced early postoperative cerebral malperfusion, however none were left with lasting neurological deficit. ${ }^{17}$ In contrast, Beckmann and colleagues reported a $21 \%(\mathrm{n}=20)$ and $26 \%(\mathrm{n}=25)$ incidence of new-onset stroke and delirium following Z-2-FET respectively, while Tsagakis et al.noted $4 \%(\mathrm{n}=8)$ of patients suffered permanent ischaemic stroke following Z-2-FET. ${ }^{12,}{ }^{22}$ This discrepancy may be due, in part, to proximalisation of the stent graft. Leontyev et al.identify distal 
landing zones as a major risk factor for SCI, and indeed Jiang et al. notes that decreased occlusion of the intercostal vessels in Z-0-FET may protect against SCI. ${ }^{18},{ }^{26}$ Rezaei et al. also highlight the use of FET stents longer than $15 \mathrm{~cm}$, or extending beyond T8, as risk factors for SCI, and that stent length less than $10 \mathrm{~cm}$ may decrease the risk of SCI. ${ }^{1}$ Jiang et al. further recommends performing FET arch repair with visceral perfusion at moderate hypothermia $\left(28^{\mathrm{O}} \mathrm{C}\right)$ to reduce overall HCA duration and attenuate risk of SCI. ${ }^{18}$ Additionally, Yamamoto and colleagues suggest that selective LSA perfusion could improve intraoperative spinal cord perfusion via collateral vasculature during HCA. ${ }^{16}$ These factors, in combination with the fact that the Z-0-FET approach involves reduced overall HCA, CPB, and ACP duration, suggest that proximalisation of FET repair may indeed reduce incidence of iatrogenic neurological injury.

\section{Renal injury}

Postoperative kidney injury frequently results from the extended durations of HCA and extracorporeal circulation often involved in complex cardiac surgeries. ${ }^{1}$ Rezaei et al.estimate that acute kidney injury (AKI) occurs in up to $40 \%$ of FET cases, while Bashir et al. reports a pooled estimate of $15.5 \%$ (95\% CI, 11.9-20.1) incidence. ${ }^{1,5}$ Factors such as advanced age, elevated preoperative serum creatinine, pre-existing hypertension, intraoperative extracorporeal circulation [?] $260 \mathrm{~min}$, CPB [?] $180 \mathrm{~min}$, and implantation of a 4branched Dacron graft with FET have been shown to increase the risk of AKI following FET arch repair. ${ }^{27,} 28$ $6 \%(n=6)$ of patients in Yamamoto and colleague's report suffered AKI and required dialysis following Z0-FET, while Jakob et al. noted a permanent renal failure and AKI incidence rates of $16.7 \% .16,1750 \%$ $(n=3)$ of patients in Jakob and colleague's trial required haemodiafiltration postoperatively. ${ }^{17}$ In contrast, the incidence of postoperative haemodiafiltration following Z-2-FET has been shown to vary between $22 \%$ and $32 \%$, and indeed Beckmann et al.highlighted $8 \%(\mathrm{n}=8)$ of their patients suffered permanent kidney failure following Z-2-FET repair. ${ }^{12,} 13,22$ It would be reasonable to suggest that that lower incidence of AKI and renal failure in Yamamoto and colleague's report could be due to the shorter duration of CPB and extracorporeal circulation associated with proximalised aortic arch repair, yet one should recall that there has yet to be any large, multi-centre studies examining postoperative outcomes from Z-0-FET. ${ }^{27}$

\section{Recurrent laryngeal nerve injury}

Proponents of zone 0 aortic arch repair argue that the proximalisation of the surgical field not only improves the surgeon's access to the sites of anastomosis but also decreases risk of damaging the recurrent laryngeal nerve $(\mathrm{RLN})$, which loops under the aortic arch in close proximity to zones 2 and $3 .{ }^{18,29}$ Indeed, Leoneet al. concluded that proximalisation of arch repair from zone 3 to zone 2 reduces the risk of RLN injury from $11 \%$ to $2.8 \%$, while Jakob and colleagues argue that Z-2-FET effectively eliminates this risk. ${ }^{18,}{ }^{23}$ It is therefore unsurprising that following Z-0-FET, Yamamoto et al. reported only a single incidence of vocal cord paralysis resulting from RLN injury. ${ }^{16}$ In contrast, Beckmann noted a 18\% ( $\mathrm{n}=17$ ) incidence of RLN palsy following Z-2-FET. ${ }^{12}$ Indeed, reduced incidence of RLN palsy may well represent surgical advancement with FET proximalisation.

\section{Reintervention}

Finally, it is worth considering the relative risk of patients having to undergo subsequent procedures following FET arch repair. One primary advantage of the FET procedure over conventional ET prosthesis is the drastically reduced incidence of reintervention after the initial procedure. ${ }^{4}$ While it is challenging to directly attribute the need for reintervention to differences between Z-0-FET and Z-2-FET, it is likely that the need for reintervention is linked to the degree of positive aortic remodelling and FL thrombosis following prosthesis implantation. ${ }^{25}$ It appears that Z-0-FET may be associated with lower rates of reintervention than Z-2-FET: while Yamamoto et al. highlight 7 cases of thoracic endovascular aortic repair (TEVAR) attributed to FL enlargement $(n=2)$, TL stenosis $(n=3)$, proximal anastomotic rupture $(n=1)$, and bowel resection $(n=1)$, $12 \%(n=19)$ of patients in Jakob and colleague's Z-2-FET report underwent aortic reintervention due to disease progression. ${ }^{16,23}$ A further $7 \%(\mathrm{n}=7)$ of patients in Beckmann's trial underwent subsequent aortic replacement. ${ }^{12}$

\section{IMPACT FOR FUTURE RESEARCH}


Though comparing the techniques and outcomes associated with Z-2-FET and Z-0-FET is vital to assess the extent to which proximalisation of arch repair represents therapeutic advancement, it should be stressed that the comparisons made herein are limited in a number of aspects. Perhaps most significantly is the fact that, relative to Z-2-FET and even Z-3-FET, Z-0-FET arch repair procedures are far more novel and are reported far less in literature. As a result, conducting statistical comparisons between the different procedures, perhaps via a meta-analysis of clinical outcomes, would be extremely challenging. Much of the literature on FET arch repairs are limited in value due to their notably small sample size and are largely retrospective cohort studies or individual case reports. ${ }^{16-18}$ Furthermore, FET arch repair procedures are particularly complex and exact surgical technique necessarily varies from patient-to-patient due to variations in anatomy, presentation, and disease progression. ${ }^{5}$ This represents an additional limitation to direct, retrospective, smaller-scale comparisons between Z-2-FET and Z-0-FET approaches. Larger scale, multi-centre, cohort studies or randomised trials including long-term follow up and monitoring would serve to elucidate better the true extent to which proximalisation of arch repair improves clinical outcomes.

\section{CONCLUSION}

The advantages of FET procedures over cET procedures, and that of Z-2-FET over Z-3-FET are widely reported and well-documented. ${ }^{1,5}$ It is clear that Z-2-FET currently represents an efficacious, robust approach to treating Type A AAD and other structural aortic arch pathologies. Indeed, recent data from novel trials investigating Z-0-FET are promising and seem to suggest that proximalisation of the distal anastomosis not only improves surgical access (thereby shortening CPB, ACP, and HCA time) but also is associated with improved mortality rates and lower incidence of SCI, cerebral injury, RLN injury, renal injury, and visceral ischaemia. ${ }^{16,17,18}$ This said, it should be recalled that efficacy of Z-0-FET in terms of FL obliteration and postoperative TL integrity is varied. ${ }^{16}$ Therefore, larger scale investigations comparing both approaches and their respective techniques and complications would shed light and help determine whether proximalisation of aortic repair from Zone 2 to Zone 0 is simply concept, or a true challenge that represents the next step forward in aortic repair.

\section{REFERENCES}

1. Rezaei Y, Bashir M, Mousavizadeh M, Daliri M, Aljadayel HA, Mohammed I, Hosseini S. Frozen elephant trunk in total arch replacement: A systematic review and meta-analysis of outcomes and aortic proximalization. Journal of Cardiac Surgery. 2021 Mar 4.

2. Tsagakis K, Wendt D, Dimitriou AM, Thielmann M, Shehada SE, El Gabry M, Jakob HG. The frozen elephant trunk treatment is the operation of choice for all kinds of arch disease. The Journal of cardiovascular surgery. 2018 May 25;59(4):540-6.

3. Borst HG. Extensive aortic replacement using" elephant trunk" prosthesis. Thorac Cardiovasc Surg. 1983;31:37-40.

4. Karck M, Chavan A, Hagl C, Friedrich H, Galanski M, Haverich A. The frozen elephant trunk technique: a new treatment for thoracic aortic aneurysms. The Journal of Thoracic and Cardiovascular Surgery. 2003 Jun 1;125(6):1550-3.

5. Choudhury RY, Basharat K, Zahra SA, Tran T, Rimmer L, Harky A, Idhrees M, Bashir M. "Proximalization is Advancement"-Zone 3 Frozen Elephant Trunk vs Zone 2 Frozen Elephant Trunk: A Literature Review. Vascular and Endovascular Surgery. 2021 Mar 23:15385744211002493.

6. Nienaber CA, Eagle KA. Aortic dissection: new frontiers in diagnosis and management: Part I: from etiology to diagnostic strategies. Circulation. 2003 Aug 5;108(5):628-35.

7. Kourliouros A, Vecht J, Kakouros N, Grapsa J, Nihoyannopoulos P, Kokotsakis J, Athanasiou T. Frozen elephant trunk as an effective alternative to open and hybrid two-stage procedures for complex aortic disease. Hellenic J Cardiol. 2011 Jul 1;52(4):337-44.

8. Bashir M, Harky A. Current status in decision making to treat acute type A dissection: limited versus extended repair. The Journal of cardiovascular surgery. 2020 Jan 23.

Rice RD, Sandhu HK, Leake SS, Afifi RO, Azizzadeh A, Charlton-Ouw KM, Nguyen TC, Miller III CC, Safi HJ, Estrera AL. Is total arch replacement associated with worse outcomes during repair of acute type 
A aortic dissection?. The Annals of thoracic surgery. 2015 Dec 1;100(6):2159-66.

Sun L, Qi R, Zhu J, Liu Y, Zheng J. Total arch replacement combined with stented elephant trunk implantation: a new "standard" therapy for type a dissection involving repair of the aortic arch?. Circulation. 2011 Mar 8;123(9):971-8.

1. Jakob H, Dohle D, Benedik J, Janosi RA, Schlosser T, Wendt D, Thielmann M, Erbel R, Tsagakis K. Long-term experience with the E-vita Open hybrid graft in complex thoracic aortic disease. European Journal of Cardio-Thoracic Surgery. 2017 Feb 1;51(2):329-38.

2. Beckmann E, Martens A, Korte W, Kaufeld T, Krueger H, Haverich A, Shrestha M. Open total arch replacement with trifurcated graft and frozen elephant trunk. Annals of cardiothoracic surgery. 2020 May;9(3):170.

3. Leone A, Di Marco L, Coppola G, Amodio C, Berardi M, Mariani C, Votano D, Bacchi Reggiani ML, Di Bartolomeo R, Pacini D. Open distal anastomosis in the frozen elephant trunk technique: initial experiences and preliminary results of arch zone 2 versus arch zone 3. European Journal of Cardio-Thoracic Surgery. 2019 Sep 1;56(3):564-71.

4. Panfilov DS, Kozlov BN, Pryakhin AS, Kopeva KV. Frozen elephant trunk technique with different proximal landing zone for aortic dissection. Interactive CardioVascular and Thoracic Surgery. 2021 Apr 13.

5. Jakob H, Idhrees M, Bashir M. From E-VITA open plus to E-VITA NEO and E-NOVIA. Journal of Cardiac Surgery. 2020 Aug 28.

6. Yamamoto H, Kadohama T, Yamaura G, Tanaka F, Takagi D, Kiryu K, Itagaki Y. Total arch repair with frozen elephant trunk using the "zone 0 arch repair" strategy for type A acute aortic dissection. The Journal of thoracic and cardiovascular surgery. 2020 Jan 1;159(1):36-45.

7. Jakob H, Shehada SE, Dohle D, Wendt D, El Gabry M, Schlosser T, Tsagakis K. New 3-zone hybrid graft: First-in-man experience in acute type I dissection. The Journal of Thoracic and Cardiovascular Surgery. 2020 May 6.

8. Jiang SM, Ali Hassan SM, Nguyen G, Bisleri G. Zone 0 frozen elephant trunk for type A retrograde acute aortic dissection following endovascular stenting of the arch. Journal of Cardiac Surgery. 2021 Feb 22 .

1. Matalanis G, Koirala RS, Shi WY, Hayward PA, McCall PR. Branch-first aortic arch replacement with no circulatory arrest or deep hypothermia. The Journal of thoracic and cardiovascular surgery. 2011 Oct 1;142(4):809-15.

2. Spielvogel D, Strauch JT, Minanov OP, Lansman SL, Griepp RB. Aortic arch replacement using a trifurcated graft and selective cerebral antegrade perfusion. The Annals of thoracic surgery. 2002 Nov $1 ; 74(5): \mathrm{S} 1810-4$.

3. Desai ND, Hoedt A, Wang G, Szeto WY, Vallabhajosyula P, Reinke M, Bavaria JE. Simplifying aortic arch surgery: open zone 2 arch with single branched thoracic endovascular aortic repair completion. Annals of cardiothoracic surgery. 2018 May;7(3):351.

Tsagakis K, Jakob H. Which frozen elephant trunk offers the optimal solution? Reflections from Essen group. InSeminars in thoracic and cardiovascular surgery 2019 Dec 1 (Vol. 31, No. 4, pp. 679-685). WB Saunders.

Jakob H, Idhrees M, Bashir M. Frozen elephant trunk with straight vascular prosthesis. Annals of cardiothoracic surgery. 2020 May;9(3):164.

1. Harky A, Fok M, Bashir M. Which is the Optimal Frozen Elephant Trunk? A Systematic Review and Meta-Analysis of Outcomes in 2161 Patients Undergoing Thoracic Aortic Aneurysm Surgery Using E-vita OPEN PLUS Hybrid Stent Graft versus Thoraflex Hybrid Prosthesis. Brazilian Journal of Cardiovascular Surgery. 2020(AHEAD).

2. Dohle DS, Tsagakis K, Janosi RA, Benedik J, Kuehl H, Penkova L, Stebner F, Wendt D, Jakob H. Aortic remodelling in aortic dissection after frozen elephant trunk. European Journal of Cardio- 
Thoracic Surgery. 2016 Jan 1;49(1):111-7.

3. Leontyev S, Tsagakis K, Pacini D, Di Bartolomeo R, Mohr FW, Weiss G, Grabenwoeger M, Mascaro JG, Iafrancesco M, Franke UF, Gobel N. Impact of clinical factors and surgical techniques on early outcome of patients treated with frozen elephant trunk technique by using EVITA open stent-graft: results of a multicentre study.

4. Shang W, Ma M, Ge YP, Liu N, Zhu JM, Sun LZ. Analysis of risk factors of type a aortic dissection (TAAD) operation of frozen elephant trunk and total arch replacement. Eur Rev Med Pharmacol Sci. 2016 Nov 1;20(21):4586-92.

5. Qiu Z, Chen L, Cao H, Zhang G, Xu F, Chen Q. Analysis of risk factors for acute kidney injury after ascending aortic replacement combined with open placement of triple-branched stent graft in type A aortic dissection: a new technique versus the traditional method in a single Chinese center. Medical science monitor: international medical journal of experimental and clinical research. 2015;21:674.

6. Moore KL, Dalley AF. Clinically oriented anatomy. Wolters Kluwer India Pvt Ltd; 2018 Jul 12.

\section{FIGURE LEGENDS}

Figure 1: Diagrammatic representations of Z-2-FET and Z-0-FET repairs using E-vita devices. The Thoraflex and Frozenix J Graft prostheses are implanted in similar ways to that which is shown above. ${ }^{15}$

Open Access Article.

Figure 2: Schematic of the surgical approach taken by Yamamoto et al. for Z-0-FET with branched aortic graft. ${ }^{16}$ BCA: brachiocephalic artery; LCA: left carotid artery; LSA: left subclavian artery; P: perfusion.

Reproduced with permission from Yamamoto H. et. al.

Figure 3: The 3-zone arch graft used by Jakob et al. in their Z-0-FET repair approach, showing the proximal cuff, uncovered arch stent, and distal covered stent. ${ }^{17}$

Reproduced with permission from Jakob $H$. et. al. 

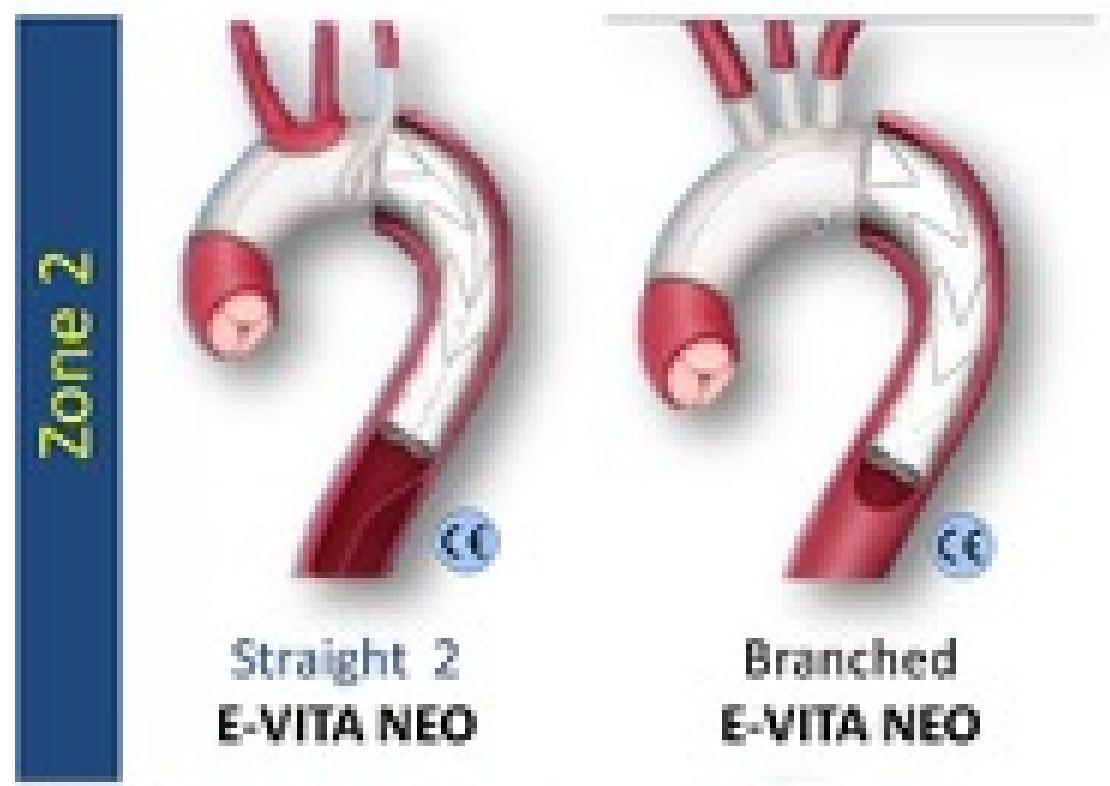

Straight 2

E-VITA NEO
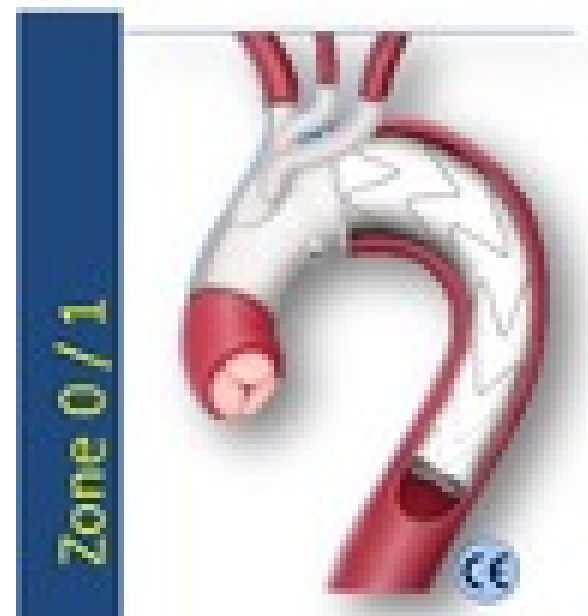

Trifurcated E-VITA NEO

\section{Branched}

E-VITA NEO

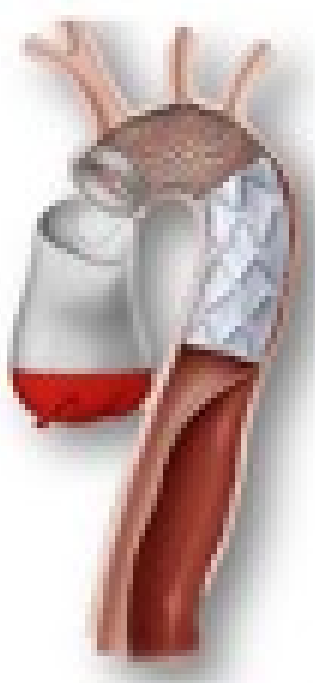

E-NOVIA 

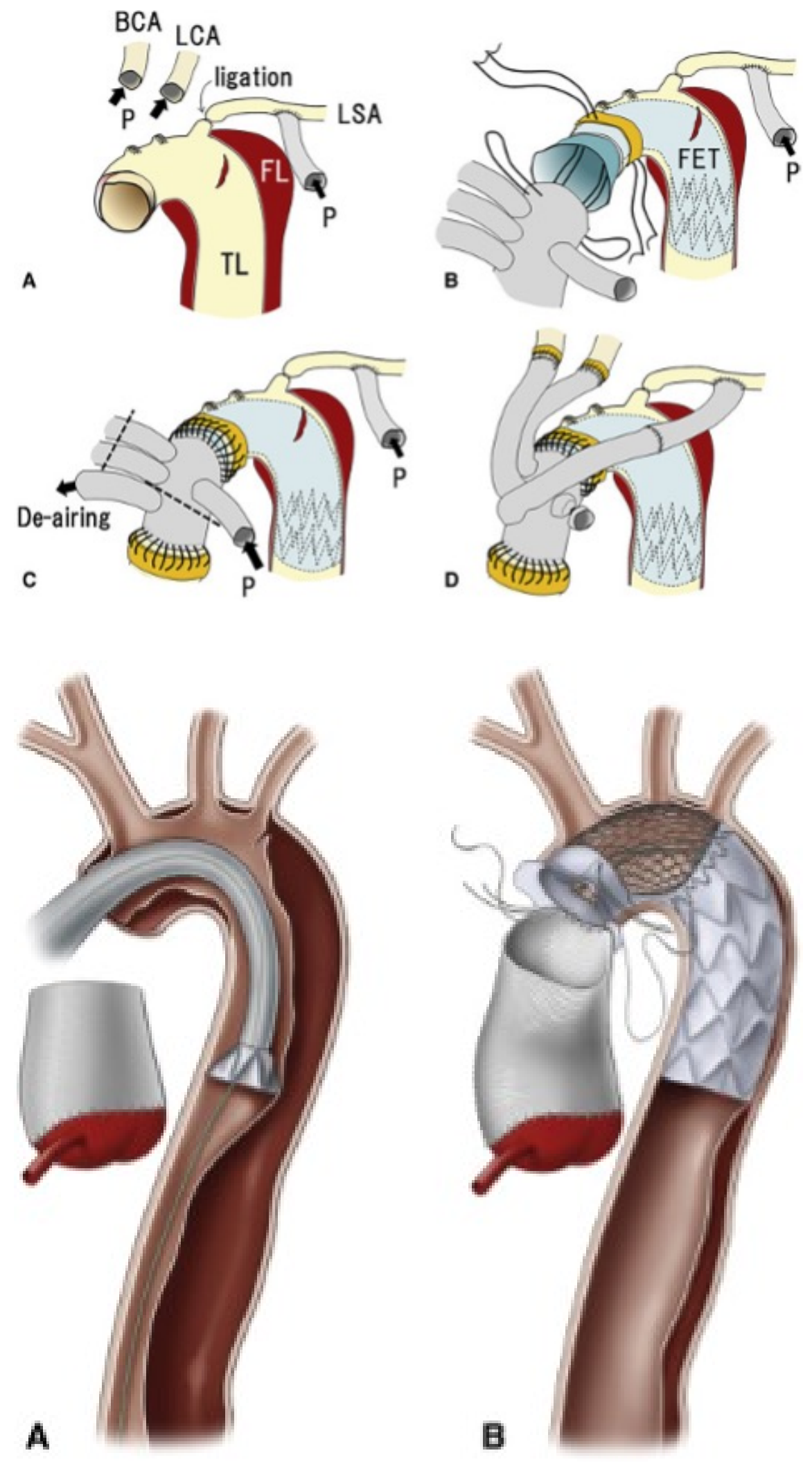